INPLASY

PROTOCOL

To cite: Ciudad et al. Gender bias in clinical trials of biological agents for severe asthma: A systematic review. Inplasy protocol 202110020. doi:

10.37766/inplasy2021.1.0020

Received: 5 January 2021

Published: 5 January 2021

Corresponding author:

Beatriz Fernández Rubio

beatrizfernandezrub@gmail.com

Author Affiliation:

Hospital Universitario Virgen

del Rocio. Avenida Manuel

Siurot $\mathbf{s} / \mathbf{n}$

Support: No financial support.

Review Stage at time of this submission: Data analysis.

Conflicts of interest:

None.

\section{Gender bias in clinical trials of biological agents for severe asthma: A systematic review}

Ciudad, P1; Fernández, B2; Guisado, AB³ .

Review question / Objective: Gender bias in clinical trials of biological agents for severe asthma.

Condition being studied: The objective of this work was to evaluate gender bias in the published CTs of biological agents for the treatment of severe asthma.

Information sources: An electronic literature search was performed using PUBMED and EMBASE.

INPLASY registration number: This protocol was registered with the International Platform of Registered Systematic Review and Meta-Analysis Protocols (INPLASY) on 5 January 2021 and was last updated on 5 January 2021 (registration number INPLASY202110020).

\section{INTRODUCTION}

Review question / Objective: Gender bias in clinical trials of biological agents for severe asthma.

Rationale: Asthma is one of the most common chronic diseases characterized by gender disparities. Gender bias is a welldocumented issue detected in the design of published clinical trials (CTs).
International guidelines encourage researchers to analyze clinical data by sex, gender, or both where appropriate Objective The objective of this work was to evaluate gender bias in the published CTs of biological agents for the treatment of severe asthma. Methods/Procedures We conducted a systematic review of randomized controlled CTs of the biological agents (omalizumab, benralizumab, reslizumab, mepolizumab or dupilumab) for 
the treatment of severe asthma. The literature search was performed using Pubmed and Embase without language restrictions. This study followed the corresponding international recommendations. Results We identified a total of $\mathbf{9 2}$ articles, of which 37 were finally included. Women represented $60.4 \%$ of patients included. The mean percentage of women in these trials was $59.9 \%$, ranged from $40.8 \%$ to $76.7 \%$. The separate analysis by sex of the main variable was only performed in five of the 37 publications included. Therefore, none of the trials analyzed secondary variables by sex. Only one of the articles discussed the results separately by sex. Conclusion The proportion of women included in CTs was higher compared to publications of other disciplines, like cancer, HIV or depression, where women were under-represented. The analysis of the main and secondary variables by sex, even the discussion separately by sex, were insufficient. This gives rise to potential gender bias in these CTs, despite the minimum requirements of international guidelines.

Condition being studied: The objective of this work was to evaluate gender bias in the published CTs of biological agents for the treatment of severe asthma.

\section{METHODS}

Search strategy: An electronic literature search was performed using PUBMED and EMBASE on May 1 2020, with no publication date or language restrictions. Search terms included a mixture of MeSH terms and free text (keywords and synonyms) combined with Boolean operators. Besides, the reference lists of selected studies were hand-searched to identify any other relevant studies.

Participant or population: 6 to 12 years (pediatrics patients) and/or $\geq 12$ years old (adults patients).

Intervention: Gender differences and in order to characterize the gender sensitivity of the trials.
Comparator: Placebo or active drug.

Study designs to be included: CTs with a control group and random assignment.

Eligibility criteria: This systematic review was carried out following the main criteria of the Preferred Reporting Items for Systematic Reviews and Meta-Analyses (PRISMA) Equity 2012 Extension declaration 15 . We selected the studies that met the following inclusion criteria: The study drug was omalizumab, benralizumab, reslizumab, mepolizumab or dupilumab. • CTs with a control group and random assignment. - Patients treated could be pediatrics or adults. - The aim of the CTs was the evaluation of the efficacy and safety of the study drug. CTs that additionally assessed other variables such as quality of life or pharmacokinetic/ pharmacodynamics were not excluded. • Patients were diagnosed with severe asthma, with an eosinophilic or allergic phenotype.

Information sources: An electronic literature search was performed using PUBMED and EMBASE.

Main outcome(s): The results of the current study show that, in general, the proportion of women included in the CTs of omalizumab, benralizumab, reslizumab, mepolizumab and dupilumab in severe asthma was higher $(60.4 \%)$ than the percentage of men.

Additional outcome(s): The CTs included showed far-from-negligible gender bias in other variables such as sex-stratification of the main and secondary outcomes, the discussion of the results analyzed by sex and the absence of the concept of "gender" in the text. Also, none of the trials followed a hormonal interaction approach to analyze the potential interaction with drugs such as hormonal contraceptives.

Quality assessment / Risk of bias analysis: Two independent reviewers (BFR and PCG) screened the titles and abstracts of all eligible publications for possible inclusion. To ensure inter-rater reliability, $100 \%$ of the 
articles were assessed independently by both authors. The articles included were full-length read before a final decision on inclusion. Any disagreement was settled by consensus with a third reviewer (ABGG).

Strategy of data synthesis: We selected the studies that met the following inclusion criteria: - The study drug was omalizumab, benralizumab, reslizumab, mepolizumab or dupilumab. • CTs with a control group and random assignment. - Patients treated could be pediatrics or adults. - The aim of the CTs was the evaluation of the efficacy and safety of the study drug. CTs that additionally assessed other variables such as quality of life or pharmacokinetic/ pharmacodynamics were not excluded. • Patients were diagnosed with severe asthma, with an eosinophilic or allergic phenotype. We excluded: • CTs in phase I. - Post-hoc analysis of one or several previous published CTs and extension CTs of previously published trials. These articles included the same patients that were evaluated in their original articles. • Systematic reviews and meta-analysis. Pilot studies with a small sample of patients. - Short reports and letters to the editor. $\bullet$ CTs that involved the evaluation of the treatment regimens based on monoclonal antibodies (mABs) plus other therapies. Those studies that allowed concomitant medications, that is, drugs that are not being studied but which a patient is taking through all or part of a study, were included.

Subgroup analysis: We also applied a subgroup analysis for the variables: date of publication, location, comparator, drug, age of patients, objectives and sample size. (all participants).

Sensibility analysis: For the analysis of gender differences and in order to characterize the gender sensitivity of the trials, we followed the Spanish recommendations for the study and evaluation of gender differences in CTs of drugs, the FDA guide, the National Institutes of Health Revitalization Act and the European Commission. In the same way, the methodology was based on the SAGER guidelines, similar recommendations published in Canada and previous publications.

Language: English or Spanish.

Country(ies) involved: Spain.

Keywords: asthma; biological agents; gender bias; sex.

Contributions of each author:

Author 1 - Pablo Ciudad Gutiérrez.

Email: pablocg95@gmail.com

Author 2 - Beatriz Fernández Rubio.

Email: beatrizfernandezrub@gmail.com

Author 3 - Ana Belén Guisado Gil.

Email: anaguigil@gmail.com 